\title{
New Results on Stability and Stabilization of Markovian Jump Systems with Time Delay
}

\author{
Hongwei Xia, ${ }^{1} \mathrm{Li}$ Li, ${ }^{2}$ Yanmin Wang, ${ }^{1}$ Guangcheng Ma, ${ }^{1}$ and Changhong Wang ${ }^{1}$ \\ ${ }^{1}$ Department of Control Science and Engineering, Harbin Institute of Technology, Harbin 150001, China \\ ${ }^{2}$ School of Information Science and Electrical Engineering, Harbin Institute of Technology at Weihai, Weihai 264200, China \\ Correspondence should be addressed to Hongwei Xia; xiahongwei@hit.edu.cn
}

Received 25 May 2014; Accepted 21 June 2014; Published 3 August 2014

Academic Editor: Ligang Wu

Copyright (c) 2014 Hongwei Xia et al. This is an open access article distributed under the Creative Commons Attribution License, which permits unrestricted use, distribution, and reproduction in any medium, provided the original work is properly cited.

\begin{abstract}
This technical paper deals with the problem of stochastic stability and stabilization for a class of linear Markovian jumping systems with discrete time-varying delay. A novel delay-dependent stochastic stability criterion for Markovian delay systems is established based on new augmented Lyapunov-Krasovskii functional and delay fractioning techniques. Then a state feedback controller is designed to guarantee the stochastic stability of the resulting closed-loop system. Numerical examples are provided to illustrate the effectiveness of the proposed design approach in this paper.
\end{abstract}

\section{Introduction}

Markovian jump systems are a special class of hybrid systems with both modes and state variables. Since many dynamical systems such as the chemical processes, transportation systems, and manufacturing systems can be modeled as Markovian jump systems, considerable attention has been devoted to the problem of analysis and synthesis for Markovian jump systems over the past decades; see, for example, [1-4] and the references therein.

On the other hand, time delays are often encountered in various practical systems such as biological, electronics, and economical systems, which are frequently one of the main causes of poor performance, oscillation, or even instability [5-10]. Thus the analysis and synthesis of Markovian jump systems with time delay are of theoretical and practical importance and have received considerable research interest in the past few decades (e.g., [1-4, 10-13]).

In the literature, different design approaches have been proposed to deal with the delays. Generally, to get stability criteria, a Lyapunov function (or functional) is involved. For more details, one can see [10-14] and references therein. In those results, the information of delay is included in some criteria, which is so-called delay dependent, while others are irrelevant to the delay [15-17], which is called delay independent. It is well known that the former ones are generally much less conservative than the latter ones due to using the information of delay such as its length or rules of changes [18-20]. Therefore, how to choose a Lyapunov function (or functional) and derive a less conservative condition from the time derivative of such a function (or functional) is one of the most important techniques in the analysis and synthesis process.

Since cross products between two vectors are commonly encountered, different bound techniques are introduced. For example, Park's inequality and Moon's inequality were frequently used for bounding some cross terms. Moreover, many researchers tried to construct special forms of Lyapunov functional to obtain a feasible result. Some delay-dependent conditions were obtained through model transformation approaches; more details can be found in [18-22] and references therein. But these choices of specific functional and overbounding techniques are the origin of conservatism [2023].

Slack matrices technique has been proven to be an effective approach to establish an improved result. To mention a few, by introducing two auxiliary matrices a new LMI representation of $\mathrm{H} \infty$ performance is presented for 
the linear systems with a single time-varying state delay in [19]. Wu et al. [20] and He et al. [21] tried to seek the relationship between the terms in the Newton-Leibniz formula and to introduce some free weighting matrices to express this relationship and some delay-dependent stability conditions with less conservativeness have been established. These methods are quite different from the conventional one of using model transformation which may introduce some superfluous dynamics into the original system. Additional studies can be found in [22-26] and references cited therein.

To improve the delay-dependent conditions, some efforts on constructing new Lyapunov functional were made by employing the delay partitioning method [21, 23-26]. To mention a few, a delay partitioning method has been developed for the delay-dependent stability analysis of fuzzy time-varying state delay systems based on a novel fuzzy Lyapunov-Krasovskii functional in [23]. In [24], by choosing an augmented Lyapunov-Krasovskii functional and utilizing the delay-partitioning method, novel delay-dependent mean square asymptotic stability conditions are derived in terms of LMIs. With the idea of delay partitioning, in [25] a new delaydependent stochastic stability criterion on the stochastic stability of Markovian jump systems with time-delay has been proposed, which is less conservative than most existing results. However, when revisiting those problems, we find that there is still much room left for improvement, which motivates the present study.

In this context, we are interested in investigating the delay-dependent stability analysis and stabilization problem for a class of Markovian jump systems with time-varying delay. By employing the delay partitioning method and introducing some slack matrix variables based on the NewtonLeibniz formula, a less conservative delay-dependent stability condition is proposed. Then a state feedback controller, which guarantees the stochastic stability of the corresponding closed-loop system, is designed.

The rest of the paper is organized as follows. Section 2 gives problem formulation and some necessary lemmas. In Section 3, we present our main results of stability analysis and stabilization problem. Numerical examples are given in Section 4, followed by the conclusions, which are presented in Section 5.

Notations. The notations used throughout the paper are standard except where otherwise stated. The superscript " $T$ " stands for matrix transposition. "I" and "0" denote the identity matrix and a zero matrix with compatible dimensions, respectively. The notation " $P>0(P \geq 0)$ " means that $P$ is real symmetric and positive definite (positive semidefinite); $\operatorname{diag}\left\{M_{1}, M_{2}, \ldots, M_{n}\right\}$ stands for a block-diagonal matrix with matrices $M_{1}, M_{2}, \ldots, M_{n}$ on the diagonal. "*" represents the elements below the main diagonal of a symmetric matrix. $\|\cdot\|$ denotes the Euclidean norm of a vector or the spectral norm of a matrix. Matrices, if their dimensions are not explicitly stated, are assumed to be compatible for algebraic operations.

\section{Problem Formulations and Preliminaries}

Consider the following stochastic linear state-delay systems with Markovian jumping parameters:

$$
\begin{gathered}
\dot{x}(t)=A(r(t)) x(t)+A_{d}(r(t)) x(t-\tau(t)) \\
+B(r(t)) u(t), \quad t>0, \\
x(t)=\varphi(t), \quad t \in[-\bar{\tau}, 0], r(0)=0,
\end{gathered}
$$

where $x(t) \in R^{n}$ is the system state vector, $u(t) \in R^{m}$ is the controlled input vector, and $A(r(t)), A_{d}(r(t))$, and $B(r(t))$ are matrix functions of the random jumping process $\{r(t)\}$. $r(t)$ is a continuous-time Markovian jump process with right continuous trajectories taking discrete values in a given finite set $S=\{1,2, \ldots, N\}$ with transition probability matrix $\Gamma \triangleq$ $\left\{\pi_{i j}\right\}$ given by

$$
\operatorname{Pr}\{r(t+\Delta)=j \mid r(t)=i\}= \begin{cases}\pi_{i j} \Delta+o(\Delta), & i \neq j \\ 1+\pi_{i j} \Delta+o(\Delta), & i=j,\end{cases}
$$

where $\Delta>0$ and $\pi_{i j} \geq 0$, for $j \neq i$, is the transition rate from mode $i$ at time $t$ to mode $j$ at time $t+\Delta$ and

$$
\pi_{i i}=-\sum_{j=1, j \neq i}^{N} \pi_{i j}
$$

for each mode $i \in S, \lim _{h \rightarrow 0}(o(\Delta) / \Delta)=0 . \varphi(t)(t \in$ $[-\bar{\tau}, 0])$ is the system's initial function which is continuous differentiable on $[-\bar{\tau}, 0]$ and $r_{0} \in S$ are the initial conditions of the continuous state and mode. $\tau(t)$ is a continuously differentiable function, which denotes the time-varying delay when the mode is in $r(t)=i$, satisfying the conditions as follows:

$$
0 \leq \tau(t) \leq \bar{\tau}, \quad \dot{\tau}(t) \leq \mu \quad \forall i \in S,
$$

where $\bar{\tau}$ and $\mu$ are known positive real constants.

Remark 1. It should be pointed out that, when a time-varying delay $\tau(t)$ is considered, it is usually assumed that $\dot{\tau}(t) \leq \mu<$ 1 , while we only require $\dot{\tau}(t) \leq \mu$ in this paper, which is more general, as discussed by $\mathrm{Xu}$ et al. [22].

For notational simplicity, in the sequel, for each possible $r(t)=i, i \in S$, a matrix $\aleph(r(t))$ will be denoted as $\aleph_{i}$; for example, $A(r(t))$ and $A_{d}(r(t))$ are denoted as $A_{i}$ and $A_{d i}$, respectively, and so on.

To facilitate further developments, we introduce the following lemma and definition that will be frequently used in deriving the main results.

Lemma 2 (Schur complement). Given constant matrices $S_{1}$, $S_{2}$, and $S_{3}$ with appropriate dimensions, where $S_{1}=S_{1}^{T}$ and $S_{2}=S_{2}^{T}>0$, then $S_{1}+S_{3}^{T} S_{2}^{-1} S_{3}<0$ if and only if

$$
\left[\begin{array}{cc}
S_{1} & S_{3}^{T} \\
* & -S_{2}
\end{array}\right]<0 \quad \text { or } \quad\left[\begin{array}{cc}
-S_{2} & S_{3}^{T} \\
* & S_{1}
\end{array}\right]<0 .
$$


Definition 3. The Markovian jump system

$$
\begin{array}{r}
\dot{x}(t)=A(r(t)) x(t)+A_{d}(r(t)) x(t-\tau(t)), \\
t>0, \\
x(t)=\varphi(t), \quad \forall t \in[-\bar{\tau}, 0], \quad r(0)=r_{0}
\end{array}
$$

is said to be stochastically stable, if for any finite $\varphi(t) \in R^{n}$ defined on $[-\bar{\tau}, 0]$ and $r_{0} \in S$ the following condition is satisfied:

$$
\lim _{t \rightarrow \infty} E\left\{\int_{0}^{t} x^{T}(t) x(t) d t \mid \varphi, r_{0}\right\}<\infty
$$

\section{Main Results}

In this section, we will consider the stability and stabilization problem of the system (1). First of all, we introduce the following results which will be used in deriving the main results.

By the Leibniz-Newton formula, we have

$$
x(t-\tau(t))=x(t)-\int_{t-\tau(t)}^{t} \dot{x}(s) d s
$$

and then for any appropriate dimensional matrices $N_{1 i}, N_{2 i}$, $N_{3 i}, N_{4 i}, N_{5 i},(i \in S)$, we have that the following equation holds:

$$
\begin{gathered}
2\left[x^{T}(t) N_{1 i}+x^{T}\left(t-\frac{\tau(t)}{2}\right) N_{2 i}+x^{T}(t-\tau(t)) N_{3 i}\right. \\
\left.+x^{T}\left(t-\frac{\tau(t)+\bar{\tau}}{2}\right) N_{4 i}+x^{T}(t-\bar{\tau}) N_{5 i}\right] \\
\quad \times\left[x(t)-x(t-\tau(t))-\int_{t-\tau(t)}^{t} \dot{x}(s) d s\right]=0 .
\end{gathered}
$$

Moreover, since $0 \leq \tau(t) \leq \bar{\tau}, \dot{\tau}(t) \leq \mu \forall i \in S$, for any appropriate dimensional matrix $X \geq 0$,

$$
\bar{\tau} \eta^{T}(t) X \eta(t)-\int_{t-\tau(t)}^{t} \eta^{T}(t) X \eta(t) d s \geq 0
$$

where

$$
\eta(t)=\left[x^{T}(t) x^{T}\left(t-\frac{\tau(t)}{2}\right) x^{T}(t-\tau(t)) x^{T}\left(t-\frac{\tau(t)+\bar{\tau}}{2}\right) x^{T}(t-\bar{\tau})\right]^{T} .
$$

3.1. Stability Analysis. Combining delay partitioning approach and free-weighting matrices technique, a new delay-dependent stochastic stability condition is established in the theorem as follows.

Theorem 4. For given positive scalars $\bar{\tau}>0$ and $\mu>0$, the autonomous Markovian jump system (1) with $u(t) \equiv 0$ is stochastically stable if there exist matrices $P_{i}=P_{i}^{T}>0$, $Q_{1 i}=Q_{1 i}^{T} \geq 0, Q_{2 i}=Q_{2 i}^{T} \geq 0, Q_{3 i}=Q_{3 i}^{T} \geq 0, Q_{4 i}=Q_{4 i}^{T} \geq 0$, $(i \in S), Z=Z^{T}>0$,

$$
X=\left[\begin{array}{ccccc}
X_{11} & X_{12} & X_{13} & X_{14} & X_{15} \\
* & X_{22} & X_{23} & X_{24} & X_{25} \\
* & * & X_{33} & X_{34} & X_{35} \\
* & * & * & X_{44} & X_{45} \\
* & * & * & * & X_{55}
\end{array}\right] \geq 0
$$

and free-weighting matrices $N_{1 i}, N_{2 i}, N_{3 i}, N_{4 i}, N_{5 i},(i \in S)$ with appropriate dimensions, such that the following LMIs hold:

$$
\Phi=\left[\begin{array}{cccccc}
\Phi_{11} & \Phi_{12} & \Phi_{13} & \Phi_{14} & \Phi_{15} & \bar{\tau} A_{i}^{T} Z \\
* & \Phi_{22} & \Phi_{23} & \Phi_{24} & \Phi_{25} & 0 \\
* & * & \Phi_{33} & \Phi_{34} & \Phi_{35} & \bar{\tau} A_{d i}^{T} Z \\
* & * & * & \Phi_{44} & \Phi_{45} & 0 \\
* & * & * & * & \Phi_{55} & 0 \\
* & * & * & * & * & -\bar{\tau} Z
\end{array}\right]<0
$$

$$
\begin{aligned}
& \Psi=\left[\begin{array}{cccccc}
X_{11} & X_{12} & X_{13} & X_{14} & X_{15} & N_{1 i} \\
* & X_{22} & X_{23} & X_{24} & X_{25} & N_{2 i} \\
* & * & X_{33} & X_{34} & X_{35} & N_{3 i} \\
* & * & * & X_{44} & X_{45} & N_{4 i} \\
* & * & * & * & X_{55} & N_{5 i} \\
* & * & * & * & * & Z
\end{array}\right] \geq 0, \\
& \sum_{j=1}^{N} \pi_{i j} Q_{1 j} \leq 0, \\
& \sum_{j=1}^{N} \pi_{i j} Q_{2 j} \leq 0, \\
& \sum_{j=1}^{N} \pi_{i j} Q_{3 j} \leq 0, \\
& \sum_{j=1}^{N} \pi_{i j} Q_{4 j} \leq 0,
\end{aligned}
$$

where

$$
\begin{aligned}
& \Phi_{11}=P_{i} A_{i}+A_{i}^{T} P_{i}+\sum_{j=1}^{N} \pi_{i j} P_{j}+Q_{1 i}+N_{1 i}+N_{1 i}^{T}+\bar{\tau} X_{11}, \\
& \Phi_{12}=N_{2 i}^{T}+\bar{\tau} X_{12},
\end{aligned}
$$




$$
\begin{aligned}
& \Phi_{13}=P_{i} A_{d i}-N_{1 i}+N_{3 i}^{T}+\bar{\tau} X_{13}, \\
& \Phi_{14}=N_{4 i}^{T}+\bar{\tau} X_{14}, \\
& \Phi_{15}=N_{5 i}^{T}+\bar{\tau} X_{15}, \\
& \Phi_{22}=-\left(1-\frac{\mu}{2}\right) Q_{1 i}+Q_{2 i}+\bar{\tau} X_{22}, \\
& \Phi_{23}=-N_{2 i}+\bar{\tau} X_{23}, \\
& \Phi_{24}=\bar{\tau} X_{24}, \\
& \Phi_{25}=\bar{\tau} X_{25}, \\
& \Phi_{33}=-(1-\mu) Q_{2 i}+Q_{3 i}-N_{3 i}-N_{3 i}^{T}+\bar{\tau} X_{33}, \\
& \Phi_{34}=-N_{4 i}^{T}+\bar{\tau} X_{34}, \\
& \Phi_{35}=-N_{5 i}^{T}+\bar{\tau} X_{35}, \\
& \Phi_{44}=-\left(1-\frac{\mu}{2}\right) Q_{3 i}+Q_{4 i}+\bar{\tau} X_{44}, \\
& \Phi_{45}=\bar{\tau} X_{45}, \\
& \Phi_{55}=-Q_{4 i}+\bar{\tau} X_{55} .
\end{aligned}
$$

Proof. We define the following Lyapunov-Krasovskii functional candidate:

$$
\begin{aligned}
V(x(t), r(t))= & V_{1}(x(t), r(t))+V_{2}(x(t), r(t)) \\
& +V_{3}(x(t), r(t))+V_{4}(x(t), r(t)) \\
& +V_{5}(x(t), r(t))+V_{6}(x(t), r(t)),
\end{aligned}
$$

with

$$
\begin{aligned}
& V_{1}(x(t), r(t))=x^{T}(t) P_{i} x(t), \\
& V_{2}(x(t), r(t))=\int_{t-(\tau(t) / 2)}^{t} x^{T}(s) Q_{1 i} x(s) d s, \\
& V_{3}(x(t), r(t))=\int_{t-\tau(t)}^{t-(\tau(t) / 2)} x^{T}(s) Q_{2 i} x(s) d s, \\
& V_{4}(x(t), r(t))=\int_{t-((\tau(t)+\bar{\tau}) / 2)}^{t-\tau(t)} x^{T}(s) Q_{3 i} x(s) d s, \\
& V_{5}(x(t), r(t))=\int_{t-\bar{\tau}}^{t-((\tau(t)+\bar{\tau}) / 2)} x^{T}(s) Q_{4 i} x(s) d s, \\
& V_{6}(x(t), r(t))=\int_{-\bar{\tau}}^{0} \int_{t+\theta}^{t} \dot{x}^{T}(s) Z \dot{x}(s) d s d \theta,
\end{aligned}
$$

where $P_{i}=P_{i}^{T}>0, Q_{1 i}=Q_{1 i}^{T} \geq 0, Q_{2 i}=Q_{2 i}^{T} \geq 0, Q_{3 i}=Q_{3 i}^{T} \geq$ $0, Q_{4 i}=Q_{4 i}^{T} \geq 0,(i \in S)$, and $Z=Z^{T}>0$ are matrices to be determined.
Let $\wp$ be the weak infinitesimal generator of the random process $\{x(t), r(t)\}$. Then, for each $r(t)=i, i \in S$, we have

$$
\begin{aligned}
& \wp V_{1}(x(t), i)=2 x^{T}(t) P_{i} \dot{x}(t)+x^{T}(t) \sum_{j=1}^{N} \pi_{i j} P_{j} x(t) \\
& =2 x^{T}(t) P_{i} A_{i} x(t)+2 x^{T}(t) P_{i} A_{d i} \\
& \times x(t-\tau(t))+x^{T}(t)\left(\sum_{j=1}^{N} \pi_{i j} P_{j}\right) x(t), \\
& \wp V_{2}(x(t), i) \\
& =x^{T}(t) Q_{1 i} x(t) \\
& -\left(1-\frac{\dot{\tau}(t)}{2}\right) x^{T}\left(t-\frac{\tau(t)}{2}\right) Q_{1 i} x\left(t-\frac{\tau(t)}{2}\right) \\
& +\int_{t-(\tau(t) / 2)}^{t} x^{T}(s)\left(\sum_{j=1}^{N} \pi_{i j} Q_{1 j}\right) x(s) d s,
\end{aligned}
$$

$\wp V_{3}(x(t), i)$

$$
\begin{aligned}
= & \int_{t-\tau(t)}^{t-(\tau(t) / 2)} x^{T}(s)\left(\sum_{j=1}^{N} \pi_{i j} Q_{2 j}\right) x(s) d s \\
& +\left(1-\frac{\dot{\tau}(t)}{2}\right) x^{T}\left(t-\frac{\tau(t)}{2}\right) Q_{2 i} x\left(t-\frac{\tau(t)}{2}\right) \\
& -(1-\dot{\tau}(t)) x^{T}(t-\tau(t)) Q_{2 i} x(t-\tau(t)),
\end{aligned}
$$

$\wp V_{4}(x(t), i)$

$$
\begin{aligned}
= & \int_{t-((\tau(t)+\bar{\tau}) / 2)}^{t-\tau(t)} x^{T}(s)\left(\sum_{j=1}^{N} \pi_{i j} Q_{3 j}\right) x(s) d s \\
& +(1-\dot{\tau}(t)) x^{T}(t-\tau(t)) Q_{3 i} x(t-\tau(t))
\end{aligned}
$$$$
-\left(1-\frac{\dot{\tau}(t)}{2}\right) x^{T}\left(t-\frac{\tau(t)+\bar{\tau}}{2}\right) Q_{3 i} x\left(t-\frac{\tau(t)+\bar{\tau}}{2}\right),
$$

$\wp V_{5}(x(t), i)$

$$
\begin{gathered}
=\left(1-\frac{\dot{\tau}(t)}{2}\right) x^{T}\left(t-\frac{\tau(t)+\bar{\tau}}{2}\right) Q_{4 i} x\left(t-\frac{\tau(t)+\bar{\tau}}{2}\right) \\
-x^{T}(t-\bar{\tau}) Q_{4 i} x(t-\bar{\tau}) \\
+\int_{t-\bar{\tau}}^{t-((\tau(t)+\bar{\tau}) / 2)} x^{T}(s)\left(\sum_{j=1}^{N} \pi_{i j} Q_{4 j}\right) x(s) d s, \\
\wp V_{6}(x(t), i)=\bar{\tau} \dot{x}^{T}(t) Z \dot{x}(t)-\int_{t-\bar{\tau}}^{t} \dot{x}^{T}(s) Z \dot{x}(s) d s .
\end{gathered}
$$

According to (8)-(11) and considering $0 \leq \tau(t) \leq \bar{\tau}, \dot{\tau}(t) \leq$ $\mu \forall i \in S$ in (4), we have

$\wp V(x(t), i)$

$$
\leq 2 x^{T}(t) P_{i} A_{i} x(t)+2 x^{T}(t) P_{i} A_{d i} x(t-\tau(t))
$$




$$
\begin{aligned}
& +x^{T}(t)\left(\sum_{j=1}^{N} \pi_{i j} P_{j}\right) x(t)+x^{T}(t) Q_{1 i} x(t) \\
& -\left(1-\frac{\bar{\tau}(t)}{2}\right) x^{T}\left(t-\frac{\tau(t)}{2}\right) Q_{1 i} x\left(t-\frac{\tau(t)}{2}\right) \\
& +\int_{t-(\tau(t) / 2)}^{t} x^{T}(s)\left(\sum_{j=1}^{N} \pi_{i j} Q_{1 j}\right) x(s) d s \\
& +\int_{t-\tau(t)}^{t-(\tau(t) / 2)} x^{T}(s)\left(\sum_{j=1}^{N} \pi_{i j} Q_{2 j}\right) x(s) d s \\
& +\left(1-\frac{\dot{\tau}(t)}{2}\right) x^{T}\left(t-\frac{\tau(t)}{2}\right) Q_{2 i} x\left(t-\frac{\tau(t)}{2}\right) \\
& -(1-\bar{\tau}(t)) x^{T}(t-\tau(t)) Q_{2 i} x(t-\tau(t)) \\
& +\int_{t-((\tau(t)+\bar{\tau}) / 2)}^{t-\tau(t)} x^{T}(s)\left(\sum_{j=1}^{N} \pi_{i j} Q_{3 j}\right) x(s) d s \\
& +(1-\dot{\tau}(t)) x^{T}(t-\tau(t)) Q_{3 i} x(t-\tau(t)) \\
& -\left(1-\frac{\bar{\tau}(t)}{2}\right) x^{T}\left(t-\frac{\tau(t)+\bar{\tau}}{2}\right) Q_{3 i} x\left(t-\frac{\tau(t)+\bar{\tau}}{2}\right) \\
& +\left(1-\frac{\dot{\tau}(t)}{2}\right) x^{T}\left(t-\frac{\tau(t)+\bar{\tau}}{2}\right) Q_{4 i} x\left(t-\frac{\tau(t)+\bar{\tau}}{2}\right) \\
& -x^{T}(t-\bar{\tau}) Q_{4 i} x(t-\bar{\tau}) \\
& +\int_{t-\bar{\tau}}^{t-((\tau(t)+\bar{\tau}) / 2)} x^{T}(s)\left(\sum_{j=1}^{N} \pi_{i j} Q_{4 j}\right) x(s) d s \\
& +\bar{\tau} \dot{x}^{T}(t) Z \dot{x}(t)-\int_{t-\bar{\tau}}^{t} \dot{x}^{T}(s) Z \dot{x}(s) d s \\
& +2\left[x^{T}(t) N_{1 i}+x^{T}\left(t-\frac{\tau(t)}{2}\right) N_{2 i}+x^{T}(t-\tau(t)) N_{3 i}\right. \\
& \left.+x^{T}\left(t-\frac{\tau(t)+\bar{\tau}}{2}\right) N_{4 i}+x^{T}(t-\bar{\tau}) N_{5 i}\right] \\
& \times\left[x(t)-x(t-\tau(t))-\int_{t-\tau(t)}^{t} \dot{x}(s) d s\right] \\
& +\bar{\tau} \eta^{T}(t) X \eta(t)-\int_{t-\tau(t)}^{t} \eta^{T}(t) X \eta(t) d s \\
& =\eta^{T}(t) \Lambda_{1} \eta(t)-\int_{t-\tau(t)}^{t} \xi^{T}(t, s) \Psi \xi(t, s) d s+\Lambda_{2}+\Lambda_{3} \text {, }
\end{aligned}
$$

where

$$
\begin{gathered}
\xi(t)=\left[\eta^{T}(t) \dot{x}^{T}(s)\right]^{T}, \\
\Lambda_{1}=\left[\begin{array}{ccccc}
\bar{\Phi}_{11} & \Phi_{12} & \bar{\Phi}_{13} & \Phi_{14} & \Phi_{15} \\
* & \Phi_{22} & \Phi_{23} & \Phi_{24} & \Phi_{25} \\
* & * & \Phi_{33} & \Phi_{34} & \Phi_{35} \\
* & * & * & \Phi_{44} & \Phi_{45} \\
* & * & * & * & \Phi_{55}
\end{array}\right],
\end{gathered}
$$

$$
\begin{gathered}
\bar{\Phi}_{11}=\Phi_{11}+A_{i}^{T} H A_{i}, \\
\bar{\Phi}_{13}=\Phi_{13}+A_{i}^{T} H A_{d i}, \\
\bar{\Phi}_{33}=\Phi_{33}+A_{d i}^{T} H A_{d i} . \\
\Lambda_{2}=\int_{t-(\tau(t) / 2)}^{t} x^{T}(s)\left(\sum_{j=1}^{N} \pi_{i j} Q_{1 j}\right) x(s) d s \\
+\int_{t-\tau(t)}^{t-(\tau(t) / 2)} x^{T}(s)\left(\sum_{j=1}^{N} \pi_{i j} Q_{2 j}\right) x(s) d s \\
+\int_{t-((\tau(t)+\bar{\tau}) / 2)}^{t-\tau(t)} x^{T}(s)\left(\sum_{j=1}^{N} \pi_{i j} Q_{3 j}\right) x(s) d s \\
+\int_{t-\bar{\tau}}^{t-((\tau(t)+\bar{\tau}) / 2)} x_{3}^{T}(s)\left(\sum_{j=1}^{N} \pi_{i j} Q_{4 j}\right) x(s) d s \\
-\frac{\dot{\tau}(t)}{2} x^{T}\left(t-\frac{\tau(t)}{2}\right) Q_{2 i} x\left(t-\frac{\tau(t)}{2}\right) \\
-\dot{\tau}(t) x^{T}(t-\tau(t)) Q_{3 i} x(t-\tau(t)) \\
\dot{\tau}(t) x^{T}\left(t-\frac{\tau(t)+\bar{\tau}}{2}\right) Q_{4 i} x\left(t-\frac{\tau(t)+\bar{\tau}}{2}\right),
\end{gathered}
$$

and $\Psi$ is denoted in (14).

By Schur complement (Lemma 2) and some matrices primary manipulations, it is easy to see that the abovementioned matrix $\Lambda_{1}<0$ in (22) is equivalent to (13).

Since $Q_{2 i} \geq 0, Q_{3 i} \geq 0, Q_{4 i} \geq 0$, we have $\Lambda_{3} \leq 0$.

It is easy to see that $\wp V(x(t), i)<0$ if LMIs (13)-(15) hold.

Set $\lambda_{1}=\min \left\{\lambda_{\min }\left(-\Lambda_{1}\right), i \in S\right\}$; then $\lambda_{1}>0$. For any $t \geq \bar{\tau}$, we have

$$
\wp V(x(t), i) \leq-\lambda_{1} \eta^{T}(t) \eta(t) \leq-\lambda_{1} x^{T}(t) x(t) .
$$

By Dynkin's formula, we have

$$
\begin{aligned}
E & \{V(x(t), i)\}-E\left\{V\left(\varphi(t), r_{0}\right)\right\} \\
& =E\left\{\int_{0}^{t} \wp V(x(s), r(s)) d s\right\} \leq-\lambda_{1} \int_{0}^{t} E\left\{x^{T}(s) x(s)\right\} d s .
\end{aligned}
$$

Hence

$$
\int_{0}^{t} E\left\{x^{T}(s) x(s)\right\} d s \leq \lambda_{1}^{-1} V\left(\varphi(t), r_{0}\right) .
$$

Set $\lambda_{2}=\min \left\{\lambda_{\min }\left(P_{i}\right), i \in S\right\}$; then $\lambda_{2}>0$. From (17), it is easy to see that for any $t \geq 0$

$$
\begin{aligned}
E\{V(x(t), i)\} \\
=E\left\{V_{1}(x(t), i)\right\}+E\left\{V_{2}(x(t), i)\right\}+E\left\{V_{3}(x(t), i)\right\} \\
\quad+E\left\{V_{4}(x(t), i)\right\}+E\left\{V_{5}(x(t), i)\right\}+E\left\{V_{6}(x(t), i)\right\} \\
\geq \\
\geq \lambda_{2} E\left\{x^{T}(t) x(t)\right\} .
\end{aligned}
$$


From inequalities (26) and (27), we know that

$$
E\left\{x^{T}(t) x(t)\right\} \leq-\chi_{1} E\left\{\int_{0}^{t} x^{T}(s) x(s) d s\right\}+\chi_{2} V\left(x_{0}, r_{0}\right),
$$

where $\chi_{1}=\lambda_{1} \lambda_{2}^{-1}, \chi_{2}=\lambda_{2}^{-1}$.

By Gronwell-Bellman lemma, we have

$$
E\left\{x^{T}(t) x(t)\right\} \leq \exp \left(-\chi_{1} t\right) \chi_{2} V\left(x_{0}, r_{0}\right) .
$$

Thus, according to (28), we have

$$
\begin{aligned}
& E\left\{\int_{0}^{t} x^{T}(s) x(s) d s \mid \varphi, r_{0}\right\} \\
& \quad \leq \chi_{1}^{-1} \chi_{2}\left(1-\exp \left(-\chi_{1} t\right)\right) V\left(x_{0}, r_{0}\right) .
\end{aligned}
$$

As $t \rightarrow \infty$, we have

$$
\lim _{t \rightarrow \infty} E\left\{\int_{0}^{t} x^{T}(s) x(s) d s \mid \varphi, r_{0}\right\} \leq \chi_{1}^{-1} \chi_{2} V\left(x_{0}, r_{0}\right) \text {. }
$$

For there always exists a scalar $\varepsilon>0$, such that

$$
\begin{aligned}
& \lim _{t \rightarrow \infty} E\left\{\int_{0}^{t} x^{T}(s) x(s) d s \mid \varphi, r_{0}\right\} \\
& \leq \chi_{1}^{-1} \chi_{2} V\left(x_{0}, r_{0}\right) \leq \varepsilon \sup _{-\bar{\tau} \leq s \leq 0}|\varphi(s)|^{2} .
\end{aligned}
$$

From Definition 3, we know that the Markovian jump system in (1) with $u(t) \equiv 0$ is stochastically stable.

The proof is thus completed.

Remark 5. Benefiting from a novel LKF construction, Theorem 4 presents a delay-dependent stochastic stability criterion which is much less conservative than most existing results in the literature. The idea of delay portioning used here is different from those approaches in the literature. For example, assuming the time delay can be denoted as a constant part and time-varying part, the idea of delay partitioning is employed to the constant part and some LKF are constructed in [21, 23-26]. As a further improved result, an appropriate LKF is established with the idea of partitioning the lower bound of time delay in [26]. However, in this brief, we divided the whole time varying delay interval $[0, \bar{\tau}]$ into multi-parts, with which a novel LKF is constructed.

If the system mode set $S=\{1\}$, the jump linear system (1) is simplified into a general linear system. Then we have the following simplified result.

Corollary 6. For given positive scalars $\bar{\tau}>0$ and $\mu>0$, the autonomous Markovian jump system (1) with $i \in S=\{1\}$ is stochastically stable if there exist matrices $P=P^{T}>0, Q_{k}=$ $Q_{k}^{T} \geq 0,(k=1,2,3,4), Z=Z^{T}>0$,

$$
X=\left[\begin{array}{ccccc}
X_{11} & X_{12} & X_{13} & X_{14} & X_{15} \\
* & X_{22} & X_{23} & X_{24} & X_{25} \\
* & * & X_{33} & X_{34} & X_{35} \\
* & * & * & X_{44} & X_{45} \\
* & * & * & * & X_{55}
\end{array}\right] \geq 0
$$

and free-weighting matrices $N_{j},(j=1,2, \ldots, 5)$ with appropriate dimensions, such that the following LMIs hold:

$\Phi=\left[\begin{array}{cccccc}\Phi_{11} & \Phi_{12} & \Phi_{13} & \Phi_{14} & \Phi_{15} & \bar{\tau} A^{T} Z \\ * & \Phi_{22} & \Phi_{23} & \Phi_{24} & \Phi_{25} & 0 \\ * & * & \Phi_{33} & \Phi_{34} & \Phi_{35} & \bar{\tau} A_{d}^{T} Z \\ * & * & * & \Phi_{44} & \Phi_{45} & 0 \\ * & * & * & * & \Phi_{55} & 0 \\ * & * & * & * & * & -\bar{\tau} Z\end{array}\right]<0$

$\Psi=\left[\begin{array}{cccccc}X_{11} & X_{12} & X_{13} & X_{14} & X_{15} & N_{1} \\ * & X_{22} & X_{23} & X_{24} & X_{25} & N_{2} \\ * & * & X_{33} & X_{34} & X_{35} & N_{3} \\ * & * & * & X_{44} & X_{45} & N_{4} \\ * & * & * & * & X_{55} & N_{5} \\ * & * & * & * & * & Z\end{array}\right] \geq 0$,

$\sum_{j=1}^{N} \pi_{i j} Q_{1} \leq 0$

$\sum_{j=1}^{N} \pi_{i j} Q_{2} \leq 0$,

$\sum_{j=1}^{N} \pi_{i j} Q_{3} \leq 0$

$$
\sum_{j=1}^{N} \pi_{i j} Q_{4} \leq 0
$$

where

$$
\begin{aligned}
& \Phi_{11}=P A+A^{T} P+Q_{1}+N_{1}+N_{1}^{T}+\bar{\tau} X_{11}, \\
& \Phi_{12}=N_{2}^{T}+\bar{\tau} X_{12}, \\
& \Phi_{13}=P A_{d}-N_{1}+N_{3}^{T}+\bar{\tau} X_{13}, \\
& \Phi_{14}=N_{4}^{T}+\bar{\tau} X_{14}, \\
& \Phi_{15}=N_{5}^{T}+\bar{\tau} X_{15}, \\
& \Phi_{22}=-\left(1-\frac{\mu}{2}\right) Q_{1}+Q_{2}+\bar{\tau} X_{22}, \\
& \Phi_{23}=-N_{2}+\bar{\tau} X_{23}, \\
& \Phi_{24}=\bar{\tau} X_{24}, \\
& \Phi_{25}=\bar{\tau} X_{25}, \\
& \Phi_{33}=-(1-\mu) Q_{2}+Q_{3}-N_{3}-N_{3}^{T}+\bar{\tau} X_{33}, \\
& \Phi_{34}=-N_{4}^{T}+\bar{\tau} X_{34}, \\
& \Phi_{35}=-N_{5}^{T}+\bar{\tau} X_{35},
\end{aligned}
$$




$$
\begin{aligned}
& \Phi_{44}=-\left(1-\frac{\mu}{2}\right) Q_{3}+Q_{4}+\bar{\tau} X_{44}, \\
& \Phi_{45}=\bar{\tau} X_{45}, \\
& \Phi_{55}=-Q_{4}+\bar{\tau} X_{55} .
\end{aligned}
$$

3.2. Stabilization Controller Design. Now we are in a position to present a solution to the stabilization problem for system (1). We assume that the state feedback controller is in the general formula as follows:

$$
u_{i}=K_{i} x(t)+K_{d i} x(t-\tau(t)),
$$

where $K_{i}$ and $K_{d i}$ are the gain matrices of the state feedback controller to be designed.

Then, for each $r(t)=i \in S$, the resulting close-loop system of (1) with (36) becomes

$$
\dot{x}(t)=\bar{A}_{i} x(t)+\bar{A}_{d i} x(t-\tau(t)),
$$

where $\bar{A}_{i}=A_{i}+B_{i} K_{i}, \bar{A}_{d i}=A_{d i}+B_{i} K_{d i}$. Thus, representing $A_{i}$ by $\bar{A}_{i}, A_{d i}$ by $\bar{A}_{d i}$ in Theorem 4 , and by some matrices primary manipulations, we can obtain the following delaydependent theorem.

Theorem 7. For given positive scalars $\bar{\tau}>0$ and $\mu>0$, the autonomous Markovian jump system (1) is stochastically stable if there exist matrices $\widetilde{P}_{i}=\widetilde{P}_{i}^{T}>0, \widetilde{Q}_{1 i}=\widetilde{Q}_{1 i}^{T} \geq 0, \widetilde{Q}_{2 i}=\widetilde{Q}_{2 i}^{T} \geq$ $0, \widetilde{Q}_{3 i}=\widetilde{Q}_{3 i}^{T} \geq 0, \widetilde{Q}_{4 i}=\widetilde{Q}_{4 i}^{T} \geq 0,(i \in S), \widetilde{Z}=\widetilde{Z}^{T}>0$,

$$
\widetilde{X}=\left[\begin{array}{ccccc}
\widetilde{X}_{11} & \widetilde{X}_{12} & \widetilde{X}_{13} & \widetilde{X}_{14} & \widetilde{X}_{15} \\
* & \widetilde{X}_{22} & \widetilde{X}_{23} & \widetilde{X}_{24} & \widetilde{X}_{25} \\
* & * & \widetilde{X}_{33} & \widetilde{X}_{34} & \widetilde{X}_{35} \\
* & * & * & \widetilde{X}_{44} & \widetilde{X}_{45} \\
* & * & * & * & \widetilde{X}_{55}
\end{array}\right] \geq 0
$$

$\widetilde{K}_{i}, \widetilde{K}_{d i}$ and free-weighting matrices $\widetilde{N}_{1 i}, \widetilde{N}_{2 i}, \widetilde{N}_{3 i}, \widetilde{N}_{4 i}, \widetilde{N}_{5 i},(i \in$ $S)$ with appropriate dimensions, such that the following LMIs hold:

$$
\widetilde{\Phi}=\left[\begin{array}{cccccc}
\widetilde{\Phi}_{11} & \widetilde{\Phi}_{12} & \widetilde{\Phi}_{13} & \widetilde{\Phi}_{14} & \widetilde{\Phi}_{15} & \widetilde{\Phi}_{16} \\
* & \widetilde{\Phi}_{22} & \widetilde{\Phi}_{23} & \widetilde{\Phi}_{24} & \widetilde{\Phi}_{25} & 0 \\
* & * & \widetilde{\Phi}_{33} & \widetilde{\Phi}_{34} & \widetilde{\Phi}_{35} & \widetilde{\Phi}_{36} \\
* & * & * & \widetilde{\Phi}_{44} & \widetilde{\Phi}_{45} & 0 \\
* & * & * & * & \widetilde{\Phi}_{55} & 0 \\
* & * & * & * & * & \widetilde{\Phi}_{66}
\end{array}\right]<0,
$$

$$
\begin{gathered}
\widetilde{\Psi}=\left[\begin{array}{cccccc}
\widetilde{X}_{11} & \widetilde{X}_{12} & \widetilde{X}_{13} & \widetilde{X}_{14} & \widetilde{X}_{15} & \widetilde{N}_{1 i} \\
* & \widetilde{X}_{22} & \widetilde{X}_{23} & \widetilde{X}_{24} & \widetilde{X}_{25} & \widetilde{N}_{2 i} \\
* & * & \widetilde{X}_{33} & \widetilde{X}_{34} & \widetilde{X}_{35} & \widetilde{N}_{3 i} \\
* & * & * & \widetilde{X}_{44} & \widetilde{X}_{45} & \widetilde{N}_{4 i} \\
* & * & * & * & \widetilde{X}_{55} & \widetilde{N}_{5 i} \\
* & * & * & * & * & \widetilde{Z}^{\prime}
\end{array}\right] \geq 0, \\
\\
\sum_{j=1}^{N} \pi_{i j} \widetilde{Q}_{1 j} \leq 0, \\
\sum_{j=1}^{N} \pi_{i j} \widetilde{Q}_{2 j} \leq 0, \\
\sum_{j=1}^{N} \pi_{i j} \widetilde{Q}_{3 j} \leq 0, \\
\sum_{j=1}^{N} \pi_{i j} \widetilde{Q}_{4 j} \leq 0,
\end{gathered}
$$

where

$$
\begin{aligned}
& \widetilde{\Phi}_{11}= A_{i} \widetilde{P}_{i}+\widetilde{P}_{i} A_{i}^{T}+B_{i} \widetilde{K}_{i}+\widetilde{K}_{i}^{T} B_{i}^{T}+\widetilde{Q}_{1 i} \\
&+\widetilde{N}_{1 i}+\widetilde{N}_{1 i}^{T}+\bar{\tau}_{11}, \\
& \widetilde{\Phi}_{12}= \widetilde{N}_{2 i}^{T}+\bar{\tau} \widetilde{X}_{12}, \\
& \widetilde{\Phi}_{13}= A_{d i} \widetilde{P}_{i}+B_{i} \widetilde{K}_{d i}-\widetilde{N}_{1 i}+\widetilde{N}_{3 i}^{T}+\bar{\tau} \widetilde{X}_{13}, \\
& \widetilde{\Phi}_{14}= \widetilde{N}_{4 i}^{T}+\bar{\tau} \widetilde{X}_{14}, \\
& \widetilde{\Phi}_{15}= \widetilde{N}_{5 i}^{T}+\bar{\tau} \widetilde{X}_{15}, \\
& \widetilde{\Phi}_{16}= \widetilde{P}_{i}^{T}+\widetilde{K}_{i}^{T} B_{i}^{T}, \\
& \widetilde{\Phi}_{22}=-\left(1-\frac{\mu}{2}\right) \widetilde{Q}_{1 i}+\widetilde{Q}_{2 i}+\bar{\tau} \widetilde{X}_{22}, \\
& \widetilde{\Phi}_{23}=-\widetilde{N}_{2 i}+\bar{\tau} \widetilde{X}_{23}, \\
& \widetilde{\Phi}_{45}= \bar{\tau} \widetilde{X}_{45}, \\
& \widetilde{\Phi}_{24}= \bar{\tau} \widetilde{X}_{24}, \\
& \widetilde{\Phi}_{25}= \bar{\tau} \widetilde{X}_{25}, \\
& \widetilde{\Phi}_{36}=-\left(\widetilde{P}_{3 i} A_{d i}^{T}+\widetilde{K}_{d i}^{T} B_{i}^{T},\right. \\
& \widetilde{\Phi}_{34}=-\widetilde{N}_{4 i}^{T}+\bar{\tau} \widetilde{X}_{34}, \\
& \widetilde{N}_{5 i}^{T}+\bar{\tau} \widetilde{X}_{35},\left.-\frac{\mu}{2}\right) \widetilde{Q}_{3 i}+\widetilde{Q}_{4 i}+\bar{\tau} \widetilde{X}_{44}, \\
& \widetilde{\Phi}_{2 i}+\widetilde{Q}_{3 i}-\widetilde{N}_{3 i}-\widetilde{N}_{3 i}^{T}+\bar{\tau} \widetilde{X}_{33}, \\
& \widetilde{\Phi}_{3 i},
\end{aligned}
$$




$$
\begin{aligned}
& \widetilde{\Phi}_{55}=-\widetilde{Q}_{4 i}+\bar{\tau} \widetilde{X}_{55}, \\
& \widetilde{\Phi}_{66}=\bar{\tau}^{-1}\left(\widetilde{Z}-2 \widetilde{P}_{i}\right) .
\end{aligned}
$$

In this case, if the conditions abovementioned are feasible, the matrices $K_{i}, K_{d i}$ in (36) are given by $K_{i}=\widetilde{K}_{i} \widetilde{P}_{i}^{-1}$ and $K_{d i}=\widetilde{K}_{d i} \widetilde{P}_{i}^{-1}$. That is, the state feedback control law (36) can be designed as

$$
u_{i}=\widetilde{K}_{i} \widetilde{P}_{i}^{-1} x(t)+\widetilde{K}_{d i} \widetilde{P}_{i}^{-1} x(t-\tau(t)) .
$$

Proof. By performing a congruence transformation $\operatorname{diag}\left\{P_{i}^{-1}, P_{i}^{-1}, P_{i}^{-1}, P_{i}^{-1}, P_{i}^{-1}\right\}$ to both sides of (12), and defining matrices $\widetilde{X}_{m n} \triangleq P_{i}^{-1} X_{m n} P_{i}^{-1},(m, n=1,2,3,4,5, i \in$ $S)$, we can obtain (38).

Similarly, by performing a congruence transformation to (14) by $\operatorname{diag}\left\{P_{i}^{-1}, P_{i}^{-1}, P_{i}^{-1}, P_{i}^{-1}, P_{i}^{-1}, P_{i}^{-1}\right\}$ and defining matrices $\widetilde{Z} \triangleq P_{i}^{-1} Z P_{i}^{-1}, \widetilde{N}_{k i} \triangleq P_{i}^{-1} N_{k i} P_{i}^{-1},(k=1,2,3,4,5, i \in S)$, we have (40).

By performing a congruence transformation to (13) with $\operatorname{diag}\left\{P_{i}^{-1}, P_{i}^{-1}, P_{i}^{-1}, P_{i}^{-1}, P_{i}^{-1}, Z^{-1}\right\}$ and defining matrices $\widetilde{P}_{i} \triangleq$ $P_{i}^{-1}, \widetilde{Q}_{k i} \triangleq P_{i}^{-1} Q_{k i} P_{i}^{-1},(k=1,2,3,4), \widetilde{K}_{i}=K_{i} P_{i}^{-1}, \widetilde{K}_{d i}=$ $K_{d i} P_{i}^{-1}$, we have

$$
\widetilde{\Phi}=\left[\begin{array}{cccccc}
\widetilde{\Phi}_{11} & \widetilde{\Phi}_{12} & \widetilde{\Phi}_{13} & \widetilde{\Phi}_{14} & \widetilde{\Phi}_{15} & \left(A_{i} \widetilde{P}_{i}+B_{i} \widetilde{K}_{i}\right)^{T} \\
* & \widetilde{\Phi}_{22} & \widetilde{\Phi}_{23} & \widetilde{\Phi}_{24} & \widetilde{\Phi}_{25} & 0 \\
* & * & \widetilde{\Phi}_{33} & \widetilde{\Phi}_{34} & \widetilde{\Phi}_{35} & \left(A_{d i} \widetilde{P}_{i}+B_{i} \widetilde{K}_{d i}\right)^{T} \\
* & * & * & \widetilde{\Phi}_{44} & \widetilde{\Phi}_{45} & 0 \\
* & * & * & * & \widetilde{\Phi}_{55} & 0 \\
* & * & * & * & * & -\bar{\tau}^{-1} \widetilde{P}_{i} \widetilde{Z}^{-1} \widetilde{P}_{i}
\end{array}\right]<0 .
$$

According to the definition of $\widetilde{P}_{i}$ and $\widetilde{Z}$, the following equation always holds:

$$
\left(\widetilde{P}_{i}-\widetilde{Z}\right)^{T} \widetilde{Z}^{-1}\left(\widetilde{P}_{i}-\widetilde{Z}\right) \geq 0,
$$

which implies that

$$
-\widetilde{P}_{i} \widetilde{Z}^{-1} \widetilde{P}_{i} \leq \widetilde{Z}-2 \widetilde{P}_{i},
$$

This completes the proof.

Remark 8. The stabilization controller design problem is solved in Theorem 7, which guarantees the addressed Markovian jump system to be stochastically stable. Sometimes, we would like to design a memory-less state feedback control law $u_{i}=K_{i} x(t)$; in this case, just set $K_{d i}=0$ in Theorem 7 .

Remark 9. It is worth pointing out that our main results are based on the LMI conditions, which can be easily obtained using LMI Control Toolbox.

\section{Numerical Examples}

In this section, some examples are presented to demonstrate the effectiveness of our proposed theoretical results.
TABLE 1: Allowable upper bound of $\bar{\tau}$ for various $\mu$.

\begin{tabular}{ccccccc}
\hline$\mu$ & 0 & 0.1 & 0.2 & 0.5 & 0.8 & 1 \\
\hline $\bar{\tau}$ & 1.3384 & 1.3224 & 1.3165 & 1.3775 & 1.5342 & 1.1651 \\
\hline
\end{tabular}

Example 1. Consider a Markovian jump system in (1) with two modes and the following parameters [27]:

$$
\begin{array}{rlrl}
A_{1} & =\left[\begin{array}{cc}
0.5 & -1 \\
0 & -3
\end{array}\right], & A_{d 1}=\left[\begin{array}{cc}
0.5 & -0.2 \\
0.2 & 0.3
\end{array}\right], \\
A_{2}=\left[\begin{array}{cc}
-5 & 1 \\
1 & 0.2
\end{array}\right], & A_{d 2}=\left[\begin{array}{cc}
-0.3 & 0.5 \\
0.4 & -0.5
\end{array}\right] .
\end{array}
$$

The generator matrix of the stochastic process $\Gamma$ is

$$
\Gamma=\left[\begin{array}{cc}
\pi_{11} & -\pi_{11} \\
-\pi_{22} & \pi_{22}
\end{array}\right] .
$$

To compare our stability result in Theorem 4 with those in [27-30], we assume $\mu=0$. When $\pi_{11}=-7$ and $\pi_{22} \geq$ -6 , the result of [28] cannot be applied to stability. When $\pi_{11}=-7$ and $\pi_{22}=-6$, based on the result of [29], the system is found to be delay-independent stable. If we increase $\pi_{22}$, for example, $\pi_{11}=-7$ and $\pi_{22}=-3$, the result of [29] cannot guarantee system stability. By the results in $[27,30]$, we can obtain a feasible solution with $\tau \leq \bar{\tau}=0.404$ and $\tau \leq \bar{\tau}=0.7316$, respectively. By Theorem 4 of this paper, we can obtain a feasible solution with $\tau \leq \bar{\tau}=1.3384$, which is much larger than that of $[27,30]$. We can see that our criterion gives a less conservative result than those in [27-30].

Example 2. Consider the Markovian jump system (1) as Example 1. For $\pi_{11}=-7$ and $\pi_{22}=-3$ and various $\mu$, the results obtained by the proposed method in Theorem 4 for the admissible upper bound value of $\bar{\tau}$ for which the system (1) remains stochastic stable are shown in Table 1.

Example 3. Consider the Markovian jump system (1) as Example 1. Suppose $\pi_{11}=-7, \pi_{22}=-3, \bar{\tau}=1.32$, and $\mu=0.1$; according to Theorem 7 , one can obtain a state feedback controller designed as

$$
u_{i}=\left\{\begin{array}{l}
{\left[\begin{array}{c}
-3.7084 \\
0.9821
\end{array}\right] x(t)+\left[\begin{array}{c}
-0.3845 \\
-0.0491
\end{array}\right] x(t-d(t)), \quad i=1} \\
{\left[\begin{array}{l}
-1.1560 \\
-0.6338
\end{array}\right] x(t)+\left[\begin{array}{c}
-0.8394 \\
0.5502
\end{array}\right] x(t-d(t)), \quad i=2 .}
\end{array}\right.
$$

The simulation results are illustrated in Figures 1-3 with the initial condition $\varphi(t)=\left[\begin{array}{ll}1 & 1\end{array}\right]^{T}\left(t \in\left[\begin{array}{ll}-\bar{\tau} & 0\end{array}\right]\right)$. Among them, Figure 1 shows the Markovian jumping signal. The control input signal $u(t)$ is given in Figure 2, while Figure 3 plots the state responses of system (1) under the presented control law $u(t)$. From the simulation, it is shown that the designed control scheme is feasible and effective since the resulting closed-loop system is stable. 


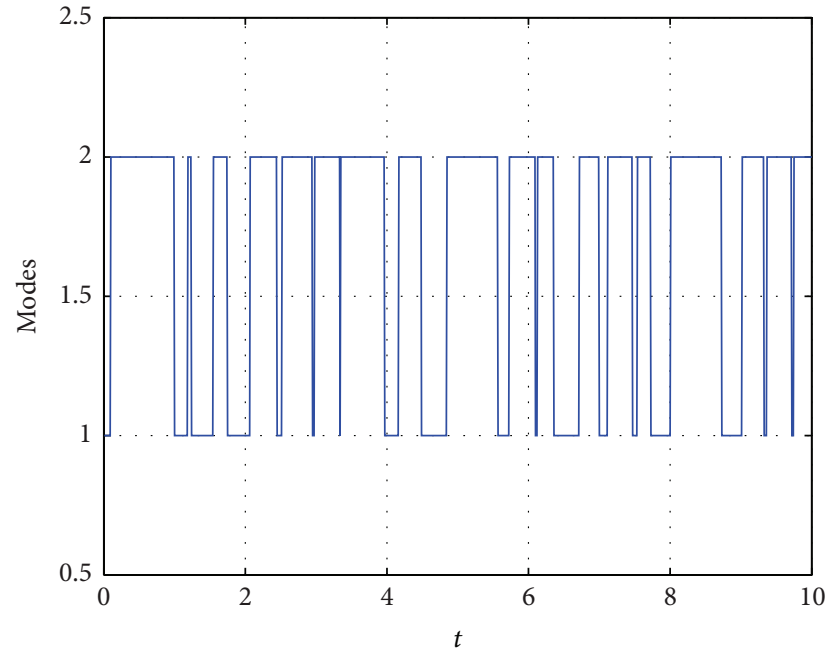

Figure 1: Markovian jumping signal.

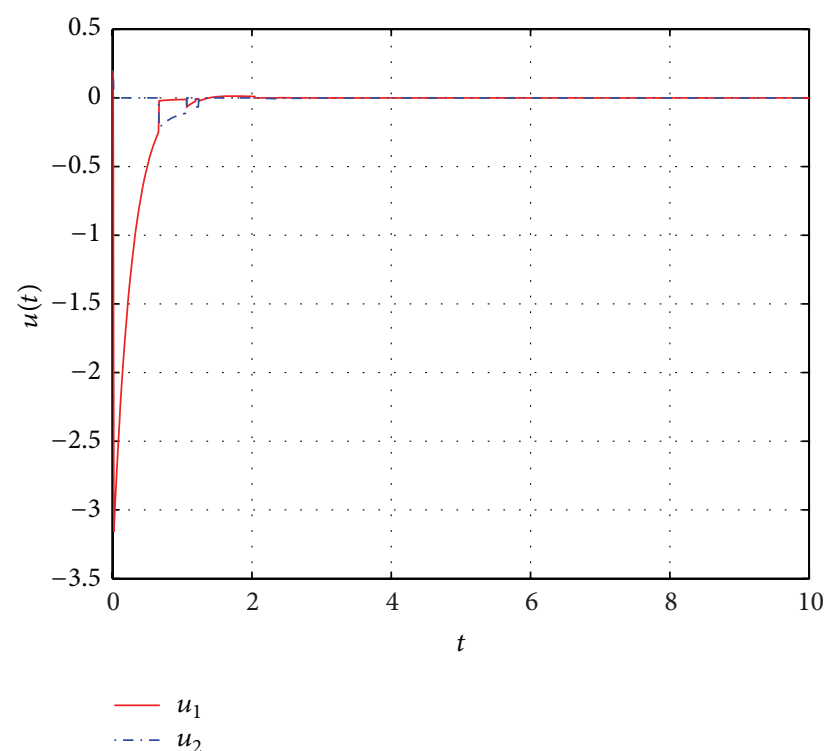

FIgURE 2: Control input.

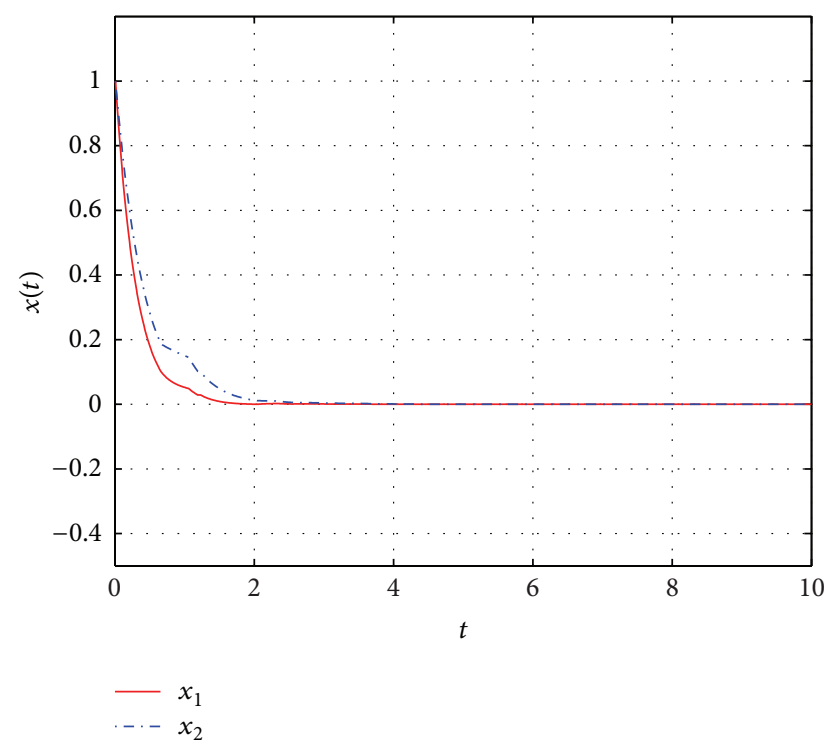

Figure 3: States of the closed-loop system.

\section{Conclusion}

In this paper, new delay-dependent conditions for stochastic stability and stabilization of a class of Markovian jump systems with time delay are presented. By the idea of delay fractioning approach and introducing some zero equations, a sufficient condition is given to guarantee the stochastic stability of the Markovian jump systems, which is less conservative than most existing results. Furthermore, based on the new criterion, an approach has been shown to design a state feedback controller, which guarantees the resulting closed-loop system to be stochastically stable. Finally, numerical examples have been provided to demonstrate the effectiveness of the proposed methods in this paper.

\section{Conflict of Interests}

The authors declare that there is no conflict of interests regarding the publication of this paper.

\section{Acknowledgments}

This work is supported by the National Natural Science Foundation of China (nos. 61304108, 61273094, and 51307035) and the Project for Distinguished Young Scholars of the Basic Research Plan in Shenzhen City under Contract no. JCJ201110001. The authors are very thankful to the reviewers for their valuable suggestions and comments.

\section{References}

[1] J. Wang, H. Wu, L. Guo, and Y. Luo, "Robust $H_{\infty}$ fuzzy control for uncertain nonlinear Markovian jump systems with timevarying delay," Fuzzy Sets and Systems, vol. 212, no. 1, pp. 41-61, 2013.

[2] L. Wu, X. Su, and P. Shi, "Sliding mode control with bounded $L_{2}$ gain performance of Markovian jump singular time-delay systems," Automatica, vol. 48, no. 8, pp. 1929-1933, 2012.

[3] U. U. Kocer, "Forecasting intermittent demand by Markov chain model," International Journal of Innovative Computing, Information and Control, vol. 9, no. 8, pp. 3307-3318, 2013.

[4] S. Long, S. Zhong, H. Zhu, and L. Xiong, "Delay-dependent stochastic admissibility for a class of discrete-time nonlinear singular Markovian jump systems with time-varying delay," Communications in Nonlinear Science and Numerical Simulation, vol. 19, no. 3, pp. 673-685, 2014.

[5] L. Wu and Z. Wang, "Guaranteed cost control of switched systems with neutral delay via dynamic output feedback," International Journal of Systems Science. Principles and Applications of Systems and Integration, vol. 40, no. 7, pp. 717-728, 2009.

[6] M. T. Alrifai, M. Zribi, M. Rayan, and M. S. Mahmoud, "On the control of time delay power systems," International Journal of Innovative Computing, Information and Control, vol. 9, no. 2, pp. 769-792, 2013.

[7] L. Wu and W. X. Zheng, "Passivity-based sliding mode control of uncertain singular time-delay systems," Automatica, vol. 45, no. 9, pp. 2120-2127, 2009. 
[8] H. K. Lam and F. H. F. Leung, "Stability analysis of discrete-time fuzzy-model-based control systems with time delay: time delayindependent approach," Fuzzy Sets and Systems, vol. 159, no. 8, pp. 990-1000, 2008.

[9] R. Yang, G. Liu, P. Shi, C. Thomas, and M. V. Basin, "Predictive output feedback control for networked control systems," IEEE Transactions on Industrial Electronics, vol. 61, no. 1, pp. 512-520, 2014.

[10] H. Li, Q. Zhou, B. Chen, and H. Liu, "Parameter-dependent robust stability for uncertain Markovian jump systems with time delay," Journal of the Franklin Institute, vol. 348, no. 4, pp. 738-748, 2011.

[11] L. Wu and W. Zheng, "Weighted $\mathrm{H}_{\infty}$ model reduction for linear switched systems with time-varying delay," Automatica, vol. 45, no. 1, pp. 186-193, 2009.

[12] X. Zhao, H. Liu, and Z. Wang, "Weighted $H_{\infty}$ performance analysis of switched linear systems with mode-dependent average dwell time," International Journal of Systems Science, vol. 44, no. 11, pp. 2130-2139, 2013.

[13] J. Lin, S. Fei, and J. Shen, "Delay-dependent $H_{\infty}$ filtering for discrete-time singular Markovian jump systems with timevarying delay and partially unknown transition probabilities," Signal Processing, vol. 91, no. 2, pp. 277-289, 2011.

[14] L. Wu, X. Su, P. Shi, and J. Qiu, "Model approximation for discrete-time state-delay systems in the TS fuzzy framework," IEEE Transactions on Fuzzy Systems, vol. 19, no. 2, pp. 366-378, 2011.

[15] F. O. Souza, M. C. de Oliveira, and R. M. Palhares, "Stability independent of delay using rational functions," Automatica, vol. 45, no. 9, pp. 2128-2133, 2009.

[16] S. Hirche, T. Matiakis, and M. Buss, "A distributed controller approach for delay-independent stability of networked control systems," Automatica, vol. 45, no. 8, pp. 1828-1836, 2009.

[17] P. A. Bliman, "LMI characterization of the strong delayindependent stability of linear delay systems via quadratic Lyapunov-Krasovskii functionals," Systems \& Control Letters, vol. 43, no. 4, pp. 263-274, 2001.

[18] E. Fridman and U. Shaked, "Delay-dependent stability and $\mathrm{H} \infty$ control: constant and time-varying delays," International Journal of Control, vol. 76, no. 1, pp. 48-60, 2003.

[19] F. Li, X. Wang, and P. Shi, "Robust quantized $H_{\infty}$ control for network control systems with Markovian jumps and time delays," International Journal of Innovative Computing, Information and Control, vol. 9, no. 12, pp. 4889-4902, 2013.

[20] M. Wu, Y. He, and J. She, "New delay-dependent stability criteria and stabilizing method for neutral systems," IEEE Transactions on Automatic Control, vol. 49, no. 12, pp. 2266-2271, 2004.

[21] Y. He, Q. Wang, L. Xie, and C. Lin, "Further improvement of free-weighting matrices technique for systems with timevarying delay," IEEE Transactions on Automatic Control, vol. 52, no. 2, pp. 293-299, 2007.

[22] S. Xu, J. Lam, and Y. Zou, "New results on delay-dependent robust $H_{\infty}$ control for systems with time-varying delays," Automatica, vol. 42, no. 2, pp. 343-348, 2006.

[23] L. Wu, X. Su, P. Shi, and J. Qiu, "A new approach to stability analysis and stabilization of discrete-time T-S fuzzy timevarying delay systems," IEEE Transactions on Systems, Man, and Cybernetics B: Cybernetics, vol. 41, no. 1, pp. 273-286, 2011.

[24] W. Chen, Q. Ma, G. Miao, and Y. Zhang, "Stability analysis of stochastic neural networks with Markovian jump parameters using delay-partitioning approach," Neurocomputing, vol. 103, pp. 22-28, 2013.

[25] Z. Fei, H. Gao, and P. Shi, "New results on stabilization of Markovian jump systems with time delay," Automatica, vol. 45, no. 10, pp. 2300-2306, 2009.

[26] H. Gao, Z. Fei, J. Lam, and B. Du, "Further results on exponential estimates of Markovian jump systems with modedependent time-varying delays," IEEE Transactions on Automatic Control, vol. 56, no. 1, pp. 223-229, 2011.

[27] J. Wu, T. Chen, and L. Wang, "Delay-dependent robust stability and $H_{\infty}$ control for jump linear systems with delays," Systems and Control Letters, vol. 55, no. 11, pp. 939-948, 2006.

[28] K. Benjelloun and E. K. Boukas, "Mean square stochastic stability of linear time-delay system with Markovian jumping parameters," IEEE Transactions on Automatic Control, vol. 43, no. 10, pp. 1456-1460, 1998.

[29] Y. Cao and J. Lam, "Robust $H \infty$ control of uncertain Markovian jump systems with time-delay," IEEE Transactions on Automatic Control, vol. 45, no. 1, pp. 77-83, 2000.

[30] Y. Cao, J. Lam, and L. Hu, "Delay-dependent stochastic stability and $H_{\infty}$ analysis for time-delay systems with Markovian jumping parameters," Journal of the Franklin Institute, vol. 340, no. 6-7, pp. 423-434, 2003. 


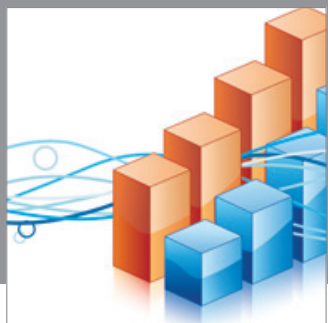

Advances in

Operations Research

mansans

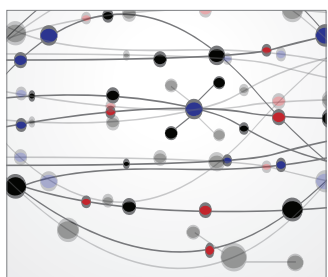

The Scientific World Journal
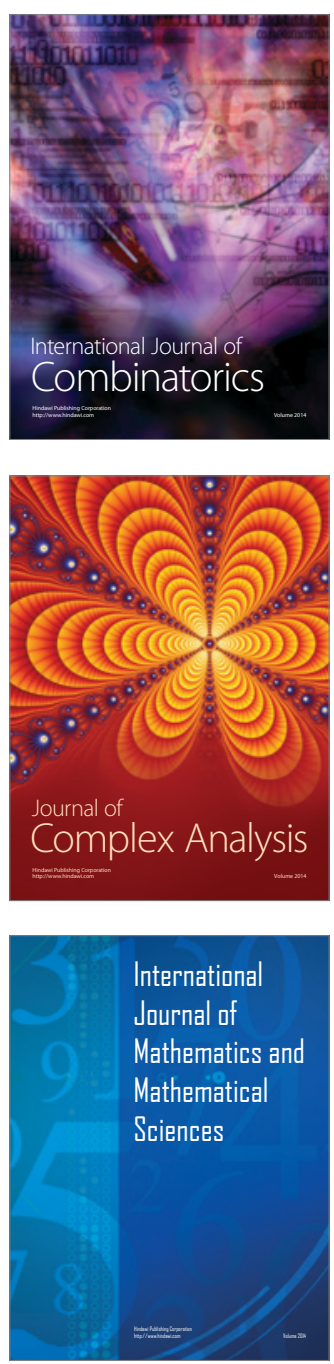
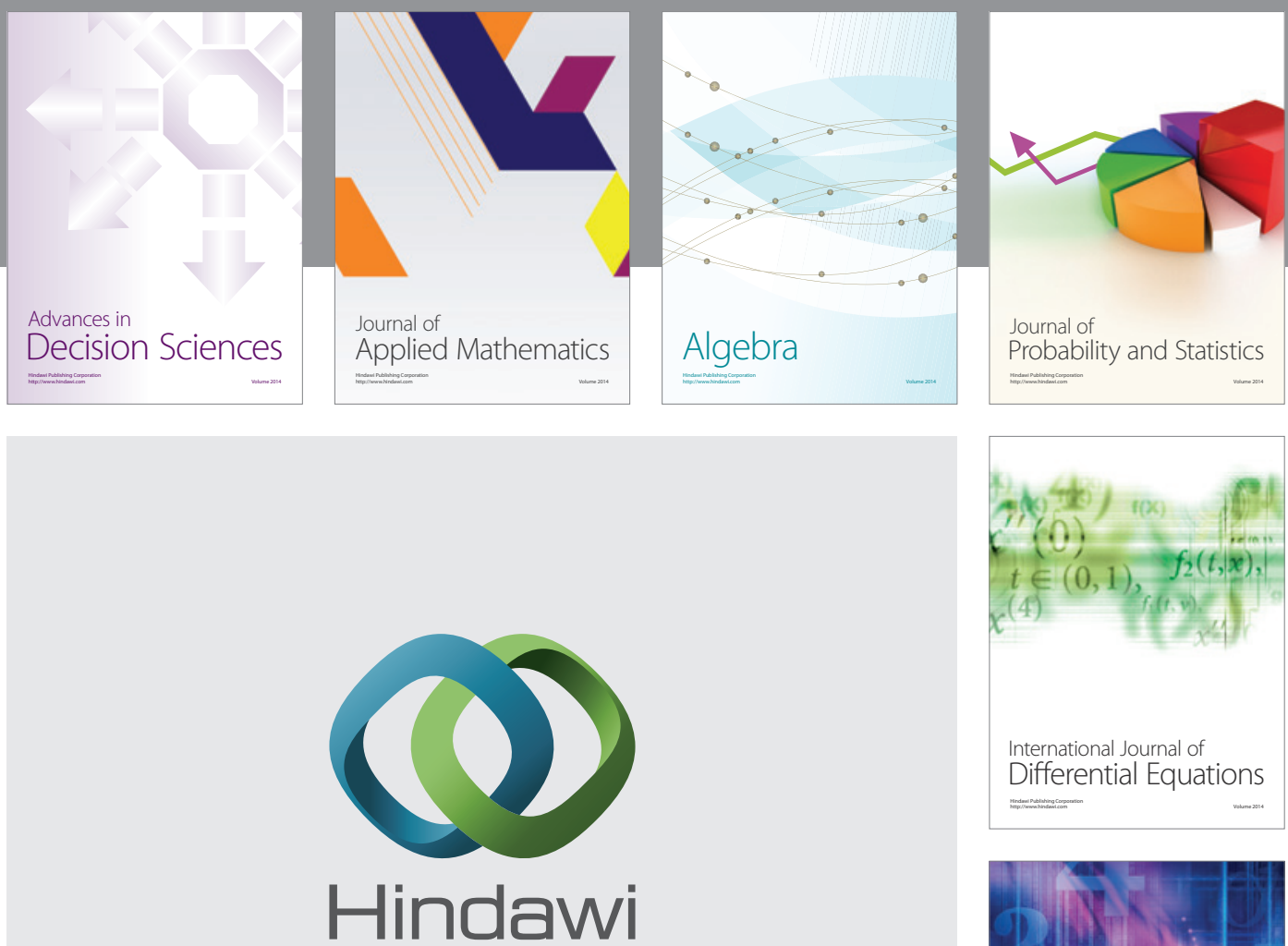

Submit your manuscripts at http://www.hindawi.com
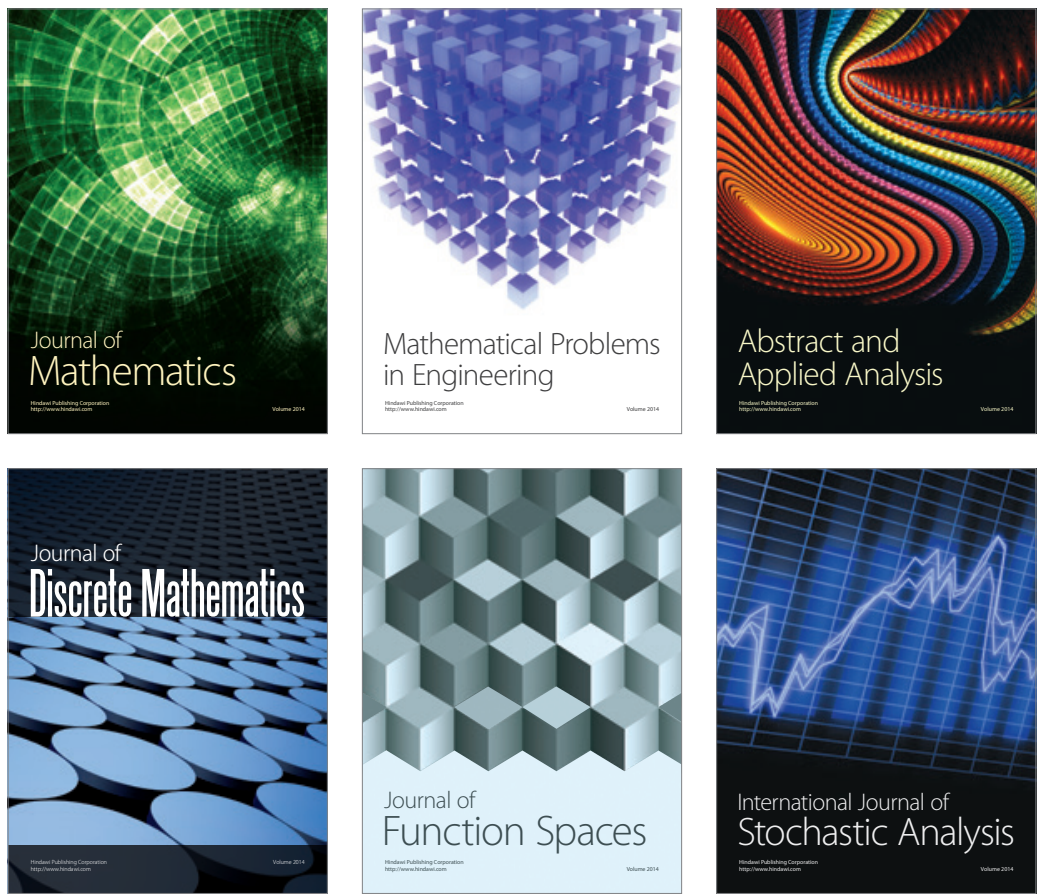

Journal of

Function Spaces

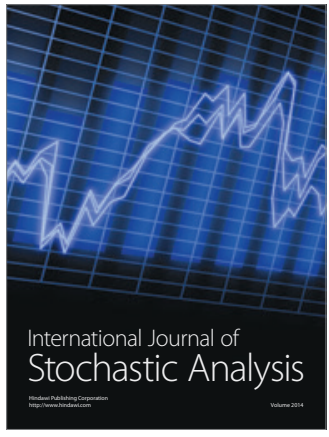

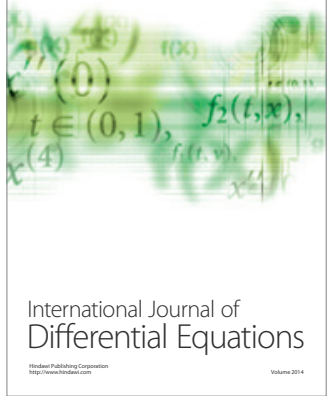
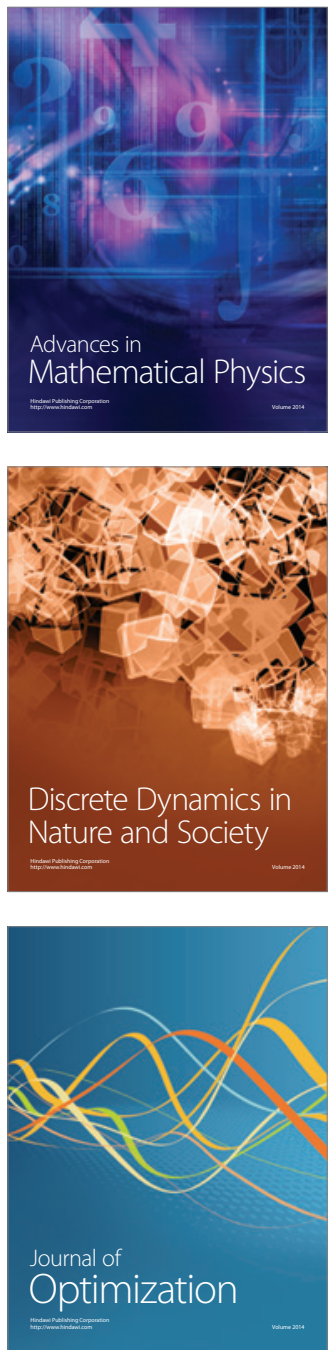